\title{
Response Prediction to Neoadjuvant Chemotherapy Prior to Interval Debulking Surgery and the Outcome of Responders Compared to Nonresponders
}

\author{
Michal Levy ${ }^{1}$, Joseph Menczer ${ }^{1}$, Mona Boaz ${ }^{2}$, Ayelet Wandel ${ }^{3}$, Yossi Mizrachi ${ }^{1}$ and Tally Levy \\ ${ }^{1}$ Department of Obstetrics and Gynecology, Division of Gynecologic Oncology, Tel Aviv University, Israel \\ ${ }^{2}$ Epidemiology and Statistics Unit, Tel Aviv University, Israel \\ ${ }^{3}$ Department of Roentgenology, Tel Aviv University, Israel
}

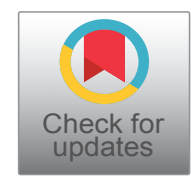

*Corresponding author: Michal Levy, MD, Gynecologic Oncology Unit, Department of Obstetrics and Gynecology, E. Wolfson Medical Center, Holon, Israel, Tel: 972-3-5028490, Fax: 972-3-5028812

\begin{abstract}
Introduction: Neoajuvant Chemotherapy (NACT) followed by Interval Debulking Surgery (IDS) is an acceptable therapeutic approach for selected patients with advanced stage Ovarian Carcinoma (OvC) and Primary Peritoneal Carcinoma (PPC) patients. Our aim was to assess whether the combined presence of reduction of the diameter of the largest tumor mass and of the CA125 level predict response to Neoadjuvant Chemotherapy (NACT).

Material and methods: Clinicopathological data were abstracted from medical records of consecutive OvC and PPC patients who received paclitaxel + carboplatin NACT and underwent IDS. Computed tomography (CT) images before NACT and prior to IDS where compared. Response to NACT prior to IDS was determined according to the combined presence of two parameters: 1 . Reduction in the greatest diameter of the largest CT tumor mass by $50 \%$ and 2. Reduction of the CA 125 level to $\leq 75 \mathrm{U} / \mathrm{ml}$. No response was determined when both of these parameters where not observed.
\end{abstract}

Results: Of 50 study group patients 10 (20.0\%) had a response and $40(80.0 \%)$ had no response to NACT. Optimal debulking $(<1 \mathrm{~cm})$ was achieved in all responders and in $92.5 \%$ of nonresponders. The median progression free survival was similar in responders and nonresponders. The Overall Survival (OS) was $62.2 \%$ in responders and $22.6 \%$ in nonresponders $(p=0.40)$. The median OS was 51 months in the nonresponders and was not reached by responders.

Conclusion: The proposed method of response prediction to NACT is simple and seems to identify patients who have a poor outcome after IDS.

\section{Keywords}

Neoadjuvant chemotherapy, Interval debulking surgery, Response, Survival

\begin{abstract}
Abbreviations
NACT: Neoadjuvant Chemotherapy; IDS: Interval Debulking Surgery; OvC: Ovarian Carcinoma; PPC: Primary Peritoneal Carcinoma; OS: Overall Survival; PFS: Progression Free Survival
\end{abstract}

\section{Key Message}

The proposed method of using the combined presence of $50 \%$ reduction in the diameter of the largest tumor mass and reduction of $\mathrm{CA} 125$ to $\leq 75$ seems to identify patients with a poor prognosis after IDS.

\section{Introduction}

Primary surgical cytoreduction followed by chemotherapy is usually the preferred management of advanced (stage III or IV) epithelial Ovarian Cancer (OvC). However it has been found that the survival of OvC patients treated by Neodjuvant Chemotherapy (NACT) followed by Interval Debulking Surgery (IDS) is not inferior to those treated by primary Debulking Surgery (PDS) $[1,2]$. Consequently, nowadays the use of NACT followed by IDS, is considered an acceptable therapeutic approach for selected patients with advanced stage OvC and Primary Peritoneal Carcinoma (PPC) patients. This approach is particularly suitable for elderly patients

Citation: Levy M, Menczer J, Boaz M, Wandel A, Mizrachi Y, et al. (2018) Response Prediction to Neoadjuvant Chemotherapy Prior to Interval Debulking Surgery and the Outcome of Responders Compared to Nonresponders. Int J Cancer Clin Res 5:098. doi.org/10.23937/2378-3419/1410098

Accepted: October 15, 2018; Published: October 17, 2018

Copyright: (C) 2018 Levy M, et al. This is an open-access article distributed under the terms of the Creative Commons Attribution License, which permits unrestricted use, distribution, and reproduction in any medium, provided the original author and source are credited. 
with bulky stage IIIC and IV disease, considered to have non resectable tumor. The aim of NACT is to reduce the tumor volume in order to facilitate optimal cytoreduction at the time of IDS.

The aim of the present study was to assess whether the combined presence of reduction of the diameter of the largest tumor mass and reduction of the CA125 level predict response to Neoadjuvant Chemotherapy (NACT) and to compare the outcome of responders to that of nonresponders after Interval Debulking Surgery (IDS).

\section{Material and Methods}

After Institutional Review Board approval (No. 020615 -WOMC of $11 / 29 / 2015)$, selected clinicopathological data were abstracted from medical records of all consecutive OvC and PPC patients who received NACT and underwent IDS diagnosed during the 12 year period between January 2004 and December 2015. The choice of NACT was based on the Nelson criteria [3]. The diagnosis of ovarian malignancy in patients receiving NACT was established by core needle biopsy or cytologic assessment of aspirated ascitic fluid. The diagnosis of PPC in these patients was made when the largest diameter of the ovaries was not greater than $3 \mathrm{~cm}$ on pretreatment imaging by transvaginal ultrasound and Computed Tomography (CT). Because the treatment and clinical course of OVC and PPC are similar they were grouped together.
Chemotherapy consisted of 3 courses of paclitaxel $175 \mathrm{mg} / \mathrm{m}^{2}+$ carboplatin AUC 6 given before and 3 courses given after IDS.

CTs where performed before NACT was given and prior to IDS. These CTs where reassessed and compared by an expert rentgenologist (A.V.). Response to NACT prior to IDS was determined according to the combined presence of two parameters: 1 . Reduction in the greatest diameter of the largest measurable tumor mass demonstrated by CT imaging by $50 \%$ and 2 . Reduction of the serum CA 125 level to $\leq 75 \mathrm{U} / \mathrm{ml}$. No response was determined when both of these parameters where not observed.

Analysis of data was carried out using SPSS v23.0 statistical analysis software (SPSS Inc., Chicago, IL, USA). Continuous variables were compared using the Mann-Whitney $U$ test. Categorical variables were compared using the chi square test or Fisher's exact test for small cells. Progression Free Survival (PFS) and Overall Survival (OS) were calculated by the Kaplan-Meier method and compared by the log-rank test.

\section{Results}

During the study period NACT was given to 50 consecutive patients (43 OvC and 7 PPC) who underwent IDS.

Table 1 presents selected characteristics of the total

Table 1: Selected characteristics of the total study group and according to response status.

\begin{tabular}{|c|c|c|c|c|c|c|c|}
\hline & \multirow{2}{*}{\multicolumn{2}{|c|}{ Total }} & \multicolumn{4}{|c|}{ Response status } & \multirow{3}{*}{ p } \\
\hline & & & \multicolumn{2}{|c|}{ Response } & \multicolumn{2}{|c|}{ No response } & \\
\hline & No. & $\%$ & No. & $\%$ & No. & $\%$ & \\
\hline Total & 50 & 100.0 & 10 & 20.0 & 40 & 80 & \\
\hline Age at diagnosis & & & & & & & 0.20 \\
\hline$\geq 65$ & 33 & 66.0 & 5 & 50.0 & 28 & 70.0 & \\
\hline$<65$ & 17 & 34.0 & 5 & 50.0 & 12 & 30.0 & \\
\hline Main complaint & & & & & & & 0.56 \\
\hline Abdominal pain & 19 & 38.0 & 6 & 60.0 & 13 & 32.5 & \\
\hline Abdominal distention & 22 & 44.0 & 3 & 30.0 & 19 & 47.5 & \\
\hline Routine examination & 6 & 12.0 & 1 & 10.0 & 5 & 12.5 & \\
\hline Other & 3 & 6.0 & 0 & 0.0 & 3 & 7.5 & \\
\hline Complaint duration (months) & & & & & & & 0.36 \\
\hline$\geq 2$ & 30 & 60.0 & 7 & 70.0 & 23 & 57.5 & \\
\hline$<2$ & 20 & 40.0 & 3 & 30.0 & 17 & 42.5 & \\
\hline Pretreatment CA125 & & & & & & & 0.24 \\
\hline$\leq 35$ & 1 & 2.0 & 1 & 10.0 & 0 & 0 & \\
\hline $36-1000$ & 23 & 46.0 & 4 & 40.0 & 19 & 47.5 & \\
\hline $1001-2000$ & 11 & 22.0 & 2 & 20.0 & 9 & 22.5 & \\
\hline$>2000$ & 15 & 30.0 & 3 & 30.0 & 12 & 30.0 & \\
\hline Stage & & & & & & & 0.57 \\
\hline III & 43 & 86.0 & 9 & 90.0 & 34 & 85.0 & \\
\hline IV & 7 & 14.0 & 1 & 10.0 & 6 & 15.0 & \\
\hline Grade & & & & & & & 0.36 \\
\hline 2 & 2 & 4.0 & 1 & 10.0 & 1 & 2.5 & \\
\hline 3 & 48 & 96.0 & 9 & 90.0 & 39 & 97.5 & \\
\hline Surgery type & & & & & & & 0.25 \\
\hline
\end{tabular}




\begin{tabular}{|l|l|l|l|l|l|l|l|}
\hline Cytoreduction & 45 & 90.0 & 8 & 80.0 & 37 & 92.5 & \\
\hline $\begin{array}{l}\text { Cytoreductive extended procedures } \\
\text { Residual disease }\end{array}$ & 5 & 10.0 & 2 & 20.0 & 3 & 7.5 & \\
\hline$<1 \mathrm{~cm}$ & & & & & & & \\
\hline$\geq 1 \mathrm{~cm}$ & 47 & 94.0 & 10 & 100.0 & 37 & 92.5 \\
\hline Postoperative complications & 3 & 6.0 & 0 & 0 & 3 & 7.5 & \\
\hline No & & & & & & & 0.41 \\
\hline Yes & 41 & 82.0 & 9 & 90.0 & 32 & 80.0 \\
\hline
\end{tabular}

Table 2: Outcome parameters in the total group and according to response status.

\begin{tabular}{|c|c|c|c|c|c|c|c|}
\hline & \multirow[b]{3}{*}{ No. } & & \multicolumn{4}{|c|}{ Response status } & \multirow[b]{3}{*}{$\mathrm{p}$} \\
\hline & & & \multicolumn{2}{|c|}{ Responders } & \multicolumn{2}{|c|}{ Nonresponders } & \\
\hline & & & No. & $\%$ & No. & $\%$ & \\
\hline Total & 50 & 100.0 & 10 & 100.0 & 40 & 100.0 & \\
\hline Residual disease & & & & & & & 0.52 \\
\hline$<1 \mathrm{~cm}$ & & & 10 & 100.0 & 37 & 92.5 & \\
\hline$\geq 1 \mathrm{~cm}$ & & & 0 & 0.0 & 3 & 7.5 & \\
\hline Median PFS, months, $(95 \% \mathrm{Cl})$ & & & $15(9.2-20.7)$ & & $14(7.3-20.6)$ & & 0.26 \\
\hline Overall survival (\%) & & & 62.2 & & 22.6 & & \\
\hline Median OS months, $(95 \% \mathrm{Cl})$ & & & Not reached & & $51(39.6-62.3)$ & & 0.79 \\
\hline
\end{tabular}

study group and according to response status. Of the 50 study group patients $10(20.0 \%)$ had a response and 40 $(80.0 \%)$ had no response to NACT according to our criteria. The majority of the patients where older than 65 years, complained of abdominal pain or distention with a duration of more than 2 months. The majority of the patients had a pretreatment CA125 level of less than $2000 \mathrm{U} / \mathrm{mL}$ had stage III grade 3 serous type tumors and all underwent cytoreductive surgery with no postoperative complications. The rate of these characteristics were similar in both response status groups.

Optimal debulking $(<1 \mathrm{~cm})$ was achieved in all $(100.0 \%)$ responders and in $92.5 \%$ of nonresponders. The median PFS was similar in responders and nonresponders 15 months (95\% Cl 9.2-20.7) and 14 months $(95 \% \mathrm{Cl} 7.3-20.6)$ respectively. The OS was $62.2 \%$ in responders and $22.6 \%$ in non responders $(p=0.40)$. The median OS was 51 months $(95 \% \mathrm{Cl} 39.6-62.3)$ in the nonresponders and the responders did not reached it (Table 2).

\section{Discussion}

We found that only $20.0 \%$ of the study group patients had a response, as defined by us, to NACT. The OS was by about $40 \%$ better in the responders than in the nonresponders and, in contrast to nonresponders, they did not reach the median OS. The lack of statistical difference in OS between responders and nonresponders and their similar PFS is most probably due to our relatively small sample size. In view of the similar PFS according to response status, the much more favorable outcome of responders found in the present study, may possibly also be due to their better response to additional lines of chemotherapy.

Our rate of optimal cytoreduction after NACT $(94.0 \%)$ is similar to that reported by others $[1,4,5]$. Interestingly there was no difference in residual disease according to response status. One could have expected a much lower rate of optimal cytoreduction in the nonresponers since their OS was much less favorable, being only $22.6 \%$. This lack of difference may again be due to our small sample size, yet it may also indicate that not only surgery affects outcome but that inherent tumor biological factors play an important prognostic role. Although many retrospective studies have shown that optimal cytoreduction confers a better treatment outcome, some have questioned its value [6]. According to a Cochrane review there is limited evidence to support the conclusion that the surgical procedure is directly responsible for the superior outcome associated with less residual disease [7]. Thus, for example, in one study [8] patients who initially presented with large-volume extrapelvic disease despite being optimally cytoreduced had worse outcomes when compared to patients who initially had small-volume disease and were also optimally cytoreduced. The authors concluded that factors other than cytoreductive surgery are important in predicting survival. In line with this are also the findings of a recent metaanalysis of 21 studies [9] that observed that optimal surgery is associated with increased PFS but it is limited to patients with less advanced disease.

In an outcome analysis of 54 OvC patients [10] the authors concluded that NACT leads to the selection of a subset of chemoresistant patients in whom aggressive surgery can be avoided since the prognosis is known to be poor regardless of treatment. The low OS survival of the nonresponders as determined by our combined criteria, indeed raises the question of whether these patients benefited from the IDS and whether they shouldn't been offered an alternative chemotherapy treatment. One option could be second line chemotherapy. However these patients may be considered as platinum resistant and in such patients the outcome after second line chemotherapy is poor with only about a 3 


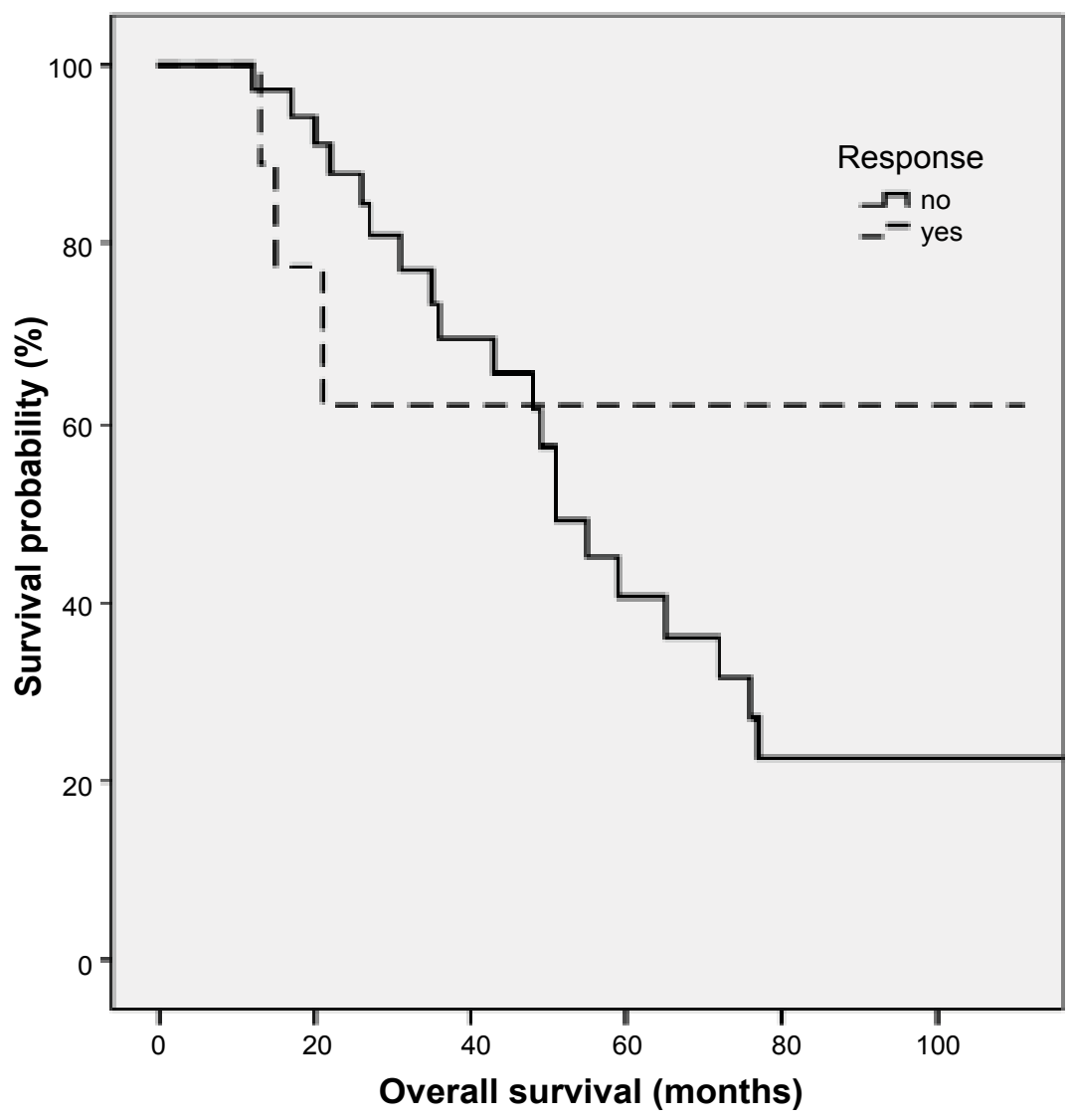

Figure 1: Survival of the study group patients according to neoadjuvant chemotherapy response status.

months duration of disease control [11]. An additional option could be the administration of more cycles of the same chemotherapy combination prior to IDS. In this context it is worthwhile to mention a study [12] that aimed to determine the efficacy and safety of six cycles of NACT with carboplatin and paclitaxel followed by IDS in 82 patients. After NACT, complete resection of all macroscopic and microscopic disease was achieved in $63.7 \%$ patients and over a median follow-up period of 19.2 months, median OS and PFS were 37.5 months and 16 months, respectively. Six cycles of NACT did not increase perioperative or postoperative complications. Another treatment option could be the addition of molecularly targeted therapy [13,14] (Figure 1).

Some studies evaluated response to NACT according to Response Evaluation Criteria in Solid Tumors (RECIST) [15]. One study [4] of 35 patients, found that among multiple parameters assessed only extensive omental disease was found to be predictive of response. Patients with extensive omental disease were more likely to have a poor response to NACT. In contrast, another study [16], based on RECIST, found that neither improvement in imaging results nor CA-125 level response prior to IDS are of prognostic value. An additional study [17] also found that lack of Ca125 response to NACT was not an independent prognostic factor. Even prospectively obtained in vitro testing data regarding platinum or paclitaxel resistance failed to be predictive of the clinical outcome in OvC treated with NACT [18].
Other studies used sequential evaluation of Positron Emission Tomography (PET) Standard Uptake Value (SUV) to predicted response to NACT. One such study [5] of 33 patients found that after the third cycle of NACT a threshold of $55 \%$ decrease in SUV optimally differentiate between responders $(n=18)$ and nonresponders $(n=15)$. The median overall survival was 38.9 months in metabolic responders and 19.7 months in nonresponders $(p=0.005)$. A similar recent study [19] of 26 patients found that the reduction in Standard Uptake Value (SUVmax) of abnormal F-DG-PET uptake evaluated before and after NACT corresponded significantly with the extent of histopathological evidence of treatment response. A cut-off value of $57 \%$ for decrease in omental SUV max was found to be able to differentiate histopathological responders from nonresponders. However, in this study, the SUVmax change was not associated with PFS.

The criterion of $50 \%$ reduction of the largest tumor diameter in our definition of response was arbitrarily chosen. The criterion of a reduction of the level of CA125 to < $75 \mathrm{U} / \mathrm{mL}$ was chosen since it has been shown that such a reduction after the 3rd NACT course was an independent predictor for complete cytoreduction at IDS [20].

The proposed method of using the combined presence of these parameters for response prediction to NACT, seems to identify patients with a poor prognosis after IDS. It is simple more accessible and less expensive than PET SUV and may therefore be more applicable. 
The disadvantages of our study are inherent in its retrospective nature and its small sample size. The advantage of our study is that the patients were treated in one institution by the same medical team. Larger studies are needed in order to confirm our results.

The authors declare that there are no conflicts of interest.

\section{References}

1. Vergote I, Tropé CG, Amant FA, Kristensen GB, Ehlen T, et al. (2010) Neoadjuvant chemotherapy or primary surgery in Stage IIlc-IV ovarian cancer. N Engl J Med 363: 943-953.

2. Tangjitgamol $S$, Manusirivithaya $S$, Laopaiboon M, Lumbiganon P, Bryant A (2016) Interval debulking surgery for advanced epithelial ovarian cancer. Cochrane Database Syst Rev 1: CD006014.

3. Nelson BE, Rosenfield AT, Schwartz PE (1993) Preoperative abdominopelvic computed tomographic prediction of optimal cytoreduction in epithelial ovarian carcinoma. J Clin Oncol 11: 166-172.

4. Yildirim Y, Ertas IE, Dogan A, Gultekin OE, Gultekin E (2012) The predictors of response to neoadjuvant chemotherapy in advanced epithelial ovarian cancer. J Surg Oncol 105: 200-205.

5. Avril N, Sassen S, Schmalfeldt B, Naehrig J, Rutke S, et al. (2005) Prediction of response to neoadjuvant chemotherapy by sequential F-18-fluorodeoxyglucose positron emission tomography in patients with advanced-stage ovarian cancer. J Clin Oncol 23: 7445-7453.

6. Covens AL (2000) A critique of surgical cytoreduction in advanced ovarian cancer. Gynecol Oncol 78: 269-274.

7. Elattar A, Bryant A, Winter-Roach BA, Hatem M, Naik R (2011) Optimal primary surgical treatment for advanced epithelial ovarian cancer. Cochrane Database 10: CD007565.

8. Hoskins WJ, Bundy BN, Thigpen JT, Omura GA (1992) The influence of cytoreductive surgery on recurrence-free interval and survival in small-volume stage III epithelial ovarian cancer: a Gynecologic Oncology Group study. Gynecol Oncol 47: 159-166.

9. Kang S, Nam BH (2009) Does neoadjuvant chemotherapy increase optimal cytoreduction rate in advanced ovarian cancer? Meta-analysis of 21 studies. Ann Surg Oncol 16: 2315-2320.

10. Ansquer $Y$, Leblanc E, Clough K, Morice P, Dauplat J, et al. (2001) Neoadjuvant chemotherapy for unresectable ovarian carcinoma: a French multicenter study. Cancer 91: 2329-2334.

11. Mutch DG, Orlando M, Goss T, Teneriello MG, Gordon AN, et al. (2007) Randomized phase III trial of gemcitabine compared with pegylated liposomal doxorubicin in patients with platinum-resistant ovarian cancer. J Clin Oncol 25: 2811-2818.

12. da Costa Miranda V, de Souza Fêde ÂB, Dos Anjos $\mathrm{CH}$, da Silva JR, Sanchez FB, et al. (2014) Neoadjuvant chemotherapy with six cycles of carboplatin and paclitaxel in advanced ovarian cancer patients unsuitable for primary surgery: Safety and effectiveness. Gynecol Oncol 132: 287-291.

13. Korkmaz T, Seber S, Basaran G (2016) Review of the current role of targeted therapies as maintenance therapies in first and second line treatment of epithelial ovarian cancer; In the light of completed trials. Crit Rev Oncol Hematol 98: 180-188.

14. Petrillo M, Nero C, Amadio G, Gallo D, Fagotti A, et al. (2016) Targeting the hallmarks of ovarian cancer: The big picture. Gynecol Oncol 142: 176-183.

15. Eisenhauer EA, Therasse P, Bogaerts J, Schwartz LH, Sargent D, et al. (2009) New response evaluation criteria in solid tumours: Revised RECIST guideline (version 1.1). Europ J Cancer 45: 228-247.

16. Menczer J, Usviatzov I, Ben-Shem E, Golan A, Levy T (2011) Neoadjuvant chemotherapy in ovarian, primary peritoneal and tubal carcinoma: can imaging results prior to interval debulking predict survival? J Gynecol Oncol 22: 183-187.

17. Le T, Hopkins L, Faught W, Fung-Kee-Fung M (2007) The lack of significance of Ca125 response in epithelial ovarian cancer patients treated with neoadjuvant chemotherapy and delayed primary surgical debulking. Gynecol Oncol 105: 712-715.

18. Tiersten AD, Moon J, Smith HO, Wilczynski SP, Robinson WR, et al. (2009) Chemotherapy resistance as a predictor of progression-free survival in ovarian cancer patients treated with neoadjuvant chemotherapy and surgical cytoreduction followed by intraperitoneal chemotherapy: a Southwest Oncology Group Study. Oncology 77: 395-399.

19. Vallius T, Peter A, Auranen A, Carpén O, Kemppainen J, et al. (2016) 18F-FDG-PET/CT can identify histopathological non-responders to platinum based neoadjuvant chemotherapy in advanced epithelial ovarian cancer. Gynecol Oncol 140: 29-35.

20. Pelissier A, Bonneau C, Chéreau E, de La Motte Rouge T, Fourchotte V Daraï E, et al. (2014) CA125 kinetic parameters predict optimal cytoreduction in patients with advanced epithelial ovarian cancer treated with neoadjuvant chemotherapy. Gynecol Oncol 135: 542-546. 